\title{
INVOLUCRAMIENTO POLÍTICO Y REPRESENTACIONES SOCIALES DE LA DEMOCRACIA EN ARGENTINA
}

\author{
ENVOLVIMENTO POLÍTICO E REPRESENTAÇÕES SOCIAIS DE \\ DEMOCRACIA NA ARGENTINA
}

\author{
POLITICAL INVOLVEMENT AND SOCIAL REPRESENTATIONS OF \\ DEMOCRACY IN ARGENTINA
}

\author{
Daniela Alonso ${ }^{12}$ e Silvina Brussino ${ }^{12}$ \\ ${ }^{1}$ Consejo Nacional de Investigaciones Científicas y Técnicas (CONICET), Córdoba, Argentina \\ ${ }^{2}$ Universidad Nacional de Córdoba (UNC), Córdoba, Argentina
}

\begin{abstract}
RESUMEN: el objetivo de este trabajo fue conocer las representaciones sociales (RS) de la democracia en función del involucramiento político. Las RS juegan un rol en la conformación de actitudes y comportamientos y pueden ayudarnos a comprender la reconocida desafección política de la ciudadanía (Dahlberg, Linde, \& Holberg, 2013). Para ello, se tomó una muestra cuotificada por sexo, edad y nivel socioeconómico de 454 personas de Córdoba (AR). Se aplicó la técnica de asociación de palabras para conocer las RS de democracia y un cuestionario cerrado para evaluar involucramiento político. Los resultados evidenciaron RS restringidas, electoralistas y liberales de la democracia. Además, la percepción de su funcionamiento real fue negativa. Los grupos que se involucraban políticamente generaron RS más sofisticadas y asignaron mayor protagonismo a la ciudadanía.
\end{abstract}

PALABRAS CLAVE: Representaciones sociales; Democracia; Sofisticación política; Participación política.

RESUMO: o objetivo deste trabalho foi conhecer as representações sociais (RS) de democracia em função do envolvimento político. As RS desempenham um papel na definição de atitudes e comportamentos e podem nos ajudar a entender o distanciamento político dos cidadãos (Dahlberg, Linde, \& Holberg, 2013). Foi realizada uma amostra cuotificada por sexo, idade e status socioeconómico de 454 cidadãos da Córdoba (AR). Para conhecer a RS da democracia aplicamos a técnica de associação de palavras e, para avaliar envolvimento político, um questionário fechado. Os resultados mostraram RS focadas em visões eleitorais restritas e liberais da democracia. Também, a percepção de seu funcionamento real foi negativa. Os grupos mais envolvidos politicamente podem gerar RS mais sofisticados e atribuir maior protagonismo à cidadania que aqueles que não estavam envueltos.

PALAVRAS-CHAVE: Representações sociais; Democracia; Sofisticação política; Participação política.

ABSTRACT: the main objective of this work was to describe the social representations (SRs) of democracy as a function of political involvement. SRs play a role in shaping attitudes and behaviors and can help us to understand the political disaffection of citizens (Dahlberg, Linde, \& Holberg, 2013). We took a sample quoted by sex, age and socioeconomic status of 454 Cordoba's (AR) citizens. The word association technique was applied to access SRs of democracy and a questionnaire to evaluate political involvement. The results showed RS centered on restricted, electoral and liberal views of democracy. In addition, the perception of its actual functioning was mainly negative. The politically involved groups generated more sophisticated SRs and assign greater protagonism to citizenship than noninvolved ones.

KEYWORDS: Social Representations; Democracy; Political Sophistication; Political Participation. 


\section{Introducción}

Las Representaciones Sociales (en adelante: RS) constituyen un conocimiento práctico que participa en la construcción social de una realidad común y sirven a la comunicación y orientación de la conducta social (Jodelet, 2011). En esta línea, las percepciones de la ciudadanía sobre el sistema democrático no son inocentes ni inocuas, sino que conllevan expectativas que afectan a la percepción y satisfacción con la democracia real, condicionando las decisiones y comportamientos políticos (Crow, 2010; Torres Stockl, \& Zubieta, 2015). Por ejemplo, si las personas tienen visiones restrictivas de la democracia (generalmente asociadas al voto) muestran mayores niveles de satisfacción que si la entienden de modo amplio (asociada a derechos y equidad social) y, a su vez, esta satisfacción afecta el compromiso cívico y la participación (Crow, 2010). En el contexto actual -y a pesar del compromiso generalizado con los valores democráticos- estos niveles de satisfacción y participación se encuentran en declive (Corporación Latinobarómetro 2015; Zovatto, 2002). Consecuentemente, resulta relevante comprender las creencias que subyacen a la adhesión a la democracia, buscando identificar algunos elementos centrales de crítica. Por esto, proponemos un análisis de las RS de la democracia argentina, procurando captar los sentidos asociados a la realidad del funcionamiento del sistema político y no solo la abstracción del concepto.

En el desarrollo de las RS intervienen aspectos culturales, ideológicos, de pertenencia social, a la vez que orientaciones afectivas, cognitivas, simbólicas y valorativas (Pérez, 2003). Consecuentemente, distintos niveles de implicación con el objeto de la RS pueden conllevar diferencias en los procesos de razonamiento social, la conformación de actitudes y comportamientos (Wachelke, 2012). Por su parte, Tessler y Gao (2008) entienden al involucramiento en función de tres elementos relacionados de la cultura cívica: el interés, el conocimiento y la participación política. Respecto del conocimiento, los estudios de RS sobre la política y la democracia señalan que la adquisición de conocimiento específico, ya sea por vía institucional -en cuyo caso se asimilarían más discursos pre-elaborados por autoridades institucionalizadas- o a través de la propia experiencia, conlleva una mayor sofisticación en las definiciones (Ruiz \& Coy, 2004; Yépez Hernández, 2003). Por otro lado, Lavine y Gschwend (2007) postulan que el procesamiento de información política requiere no sólo habilidad (conocimiento) sino también motivación; así, conocimiento político e interés son indicadores del desarrollo de los esquemas políticos (Rhee \& Capella, 1997) y elementos de la sofisticación política (en adelante: SP) en sus dimensiones cognitivas y motivacionales (Weisber \& Nawara, 2010). Por su parte, la participación política y cívica no sólo es un indicador de implicación política, sino también un espacio de socialización que fomenta el desarrollo de valores, conocimientos y habilidades útiles para el ejercicio de la ciudadanía democrática (Tessler \& Gao, 2008). Consecuentemente, recuperamos aquí el rol de estos dos indicadores de implicación política (SP y participación) en el desarrollo de las RS sobre democracia.

Respecto del contenido de las nociones de democracia, retomamos la categorización de Crow (2010), reproducida - con variaciones- en mucha de la literatura sobre RS de democracia (Bermúdez, Savino, \& Zebklussen, 2004; Dalton, Sin, \& Jou 2007), que nos permite identificar definiciones restrictivas y sustantivas de este concepto. La definición más restrictiva es la de democracia electoral, como mecanismo de elección de representantes y acotada al ámbito de la autoridad política. Esta se corresponde parcialmente con definiciones procedimentales, dado que ambas excluyen mecanismos de acción no formales y aspec- 
tos sustantivos del bienestar social. Desde un punto de vista empírico, recoge definiciones vinculadas al voto, las elecciones y el sistema multipartidario (Bermúdez et al., 2004; Crow, 2010; Dalton et al., 2007). Por su parte, la noción de democracia liberal -enraizada en el liberalismo del siglo XIX- adiciona elementos que remiten a valores; vinculados a derechos, libertades e igualdad. Sin embargo -por su raíz liberal- suele reflejar visiones individualistas del orden social (por ejemplo, igualdad ante la ley antes que igualdad social) (Moodie, Marková, \& Plichtová, 1995). En el trabajo de Dalton et al. (2007) una noción cercana a esta es la de democracia definida por resultados, en la que estos valores representan resultados deseables de la institucionalidad democrática. En cualquier caso, es relativamente más amplia que la anterior al incluir constricciones al poder político y rendición de cuentas horizontal y vertical (Crow, 2010). Finalmente, la democracia sustantiva es para nosotros la visión más amplia, en tanto señala a la justicia social como objetivo último de la democracia. Así, descansa en una idea de ciudadanía social que implica el derecho de todos y todas de compartir los beneficios de la sociedad. Estas definiciones conllevan ampliación de derechos, incluyendo educación, salud, trabajo; siendo el estado garante de ellos (Dalton et al., 2007). Por su parte, Bermúdez et al. (2004) incluyen también aspectos culturales y de participación de la sociedad civil; mientras que Dalton et al. (2007) la entiende como indicador aspiracional de sociedades menos desarrolladas, posicionando a las definiciones liberales como expresión deseable de una conciencia democrática.

Estos tipos ideales se reproducen en las asociaciones y discursos de la ciudadanía; donde se corrobora la supremacía de un enfoque liberal y restrictivo de la democracia, basado casi exclusivamente en libertades individuales e igualdad ante la ley. Estudios en países de Europa Central y Occidental, así como de democracias con menos trayectoria y desarrollo arriban a conclusiones similares: predominan las RS vinculadas a una noción liberal representativa de democracia -principalmente la libertad e igualdad-, siendo más frecuentes que las referencias restringidas al electoralismo; aunque estas últimas son relativamente más habituales en países con menor trayectoria democrática (Dalton et al., 2007; Magioglou, 2000; Moodie et al., 1995; Staerkle et al., 2015). Los estudios en la región latinoamericana van en el mismo sentido: la noción latinoamericana de democracia se centra en la libertad, la justicia y la igualdad (democracia liberal) y, en menor medida, en las elecciones (democracia electoral) (Bruno \& Barreiro, 2015; González Aguilar, 2016; Cerda, Herrera, Moreno, \& Romero, 2016; Ruiz \& Coy, 2004; Yépez Hernández, 2003; Zovatto, 2002). En el caso de la Ciudad de Córdoba, Bermúdez et al. (2004), en un estudio cualitativo con adolescentes, encontraron elementos de democracia sustantiva-satisfacción de las necesidades de la ciudadanía como condición para una democracia plena-, aunque igualmente prevalecieron los conceptos liberales -libertad e igualdad ante la ley-.

Existe otro eje que atraviesa los estudios sobre RS sobre democracia vinculado a la distancia entre las definiciones utópicas/ideales de la democracia (el deber ser) y las que remiten a la aplicación real de la misma (el ser real) (Torres Stockl \& Zubieta, 2015). En la práctica, esta diferenciación suele corresponderse con la que hay entre las visiones positivas (deber ser) y negativas (real) (Echebarría \& Álvarez, 1996; González Aguilar, 2016; Lozada, 1997; Magioglou, 2000; Cerda et al., 2016; Yépez Hernández, 2003). Por ejemplo, numerosos estudios -principalmente en el contex to latinoamericano- identifican elementos centrales negativos, entre los que se destacan aquellos relativos a la manipulación, la mentira y la represión (Echebarría \& Álvarez, 1996), así como al incumplimiento de promesas electorales y una alienación generalizada respecto de la clase política basada 
en la corrupción y el clientelismo (Bermúdez et al,, 2004). Así, mucho de estos contenidos remiten a actores centrales de la democracia como los partidos políticos (González Aguilar, 2016). Esto estaría en la base de lo que González Aguilar denomina paradoja de la democracia actual: la coexistencia de un ambiente con expectativas continuas de cambio político y el desencanto y pesimismo respecto de la posibilidad de verlo realizado. Por su parte, Lozada (1997) en un estudio cualitativo con población venezolana, suma una tercera dimensión: la democracia prospectivo-ideal. Así, podríamos identificar los elementos ideales (deber ser), los reales (ser real) y los posibles: la democracia prospectivo-ideal es la que es pasible de ser construida a partir de los vestigios de la real y lo realizable de la teórico-ideal. Según esta autora, el camino principal para ello sería la participación y el involucramiento de los sectores hoy excluidos. Complementariamente, Cerda et al. (2016) señalan la pluralidad, participación y expresión como elementos del enlace entre lo simbólico-ideal y lo real; atribuyendo el potencial de cambio al involucramiento cívico más que a los canales institucionales de la democracia.

En conjunto, los resultados -si bien replican la polisemia del concepto- señalan más similitudes que diferencias en los distintos contextos, aunque el hecho de estar centrados mayoritariamente en población joven y con acceso a la educación superior (la mayoría de las muestras son de estudiantes secundarios o universitarios), puede atentar contra la posibilidad de generalizar los resultados a la población general. Este es un aspecto que contemplamos aquí al incluir población de todas las edades y segmentos socioeconómicos. Además, no registramos antecedentes que evaluaran explícitamente el involucramiento político y examinaran su rol respecto de las RS. En consecuencia, nuestro objetivo es explorar el contenido y estructura de las RS de la democracia argentina en función del grado de involucramiento político -entendido en términos de participación y sofisticación política-y, a partir de ello, identificar las diferencias entre éstas. En términos generales, la evidencia sugiere la posibilidad de encontrar un núcleo representacional relativamente estable, asociado a una RS hegemónica de la democracia liberal y procedimental. Además, se espera un contenido polarizante entre el deber ser (positivo) y el ser real (negativo) y una mayor sofisticación de las RS en los grupos más implicados políticamente.

\section{Método y Materiales}

Se escogió un diseño de investigación descriptivo dado que nuestro objetivo central fue describir cómo se manifiesta un fenómeno en función de la especificación de sus propiedades relevantes (Hernández Sampieri, Fernández Collado, \& Baptista Lucio, 1991). Asimismo, se utilizaron técnicas cualitativas y cuantitativas para la recolección y análisis de la información.

\subsection{Población y muestra}

La población en estudio fueron ciudadanos/as de Córdoba entre 18 y 70 años. La muestra quedó compuesta por 450 personas con una distribución similar a la población general en términos de características sociodemográficas. Se aplicó un muestreo no probabilístico por cuotas de sexo, edad y nivel socioeconómico (en adelante: NSE) generadas en función de datos de la encuesta anual de hogares urbanos del INDEC (Muraro, 2012). El 
$52,6 \%$ fueron mujeres y la media de edad de 37,3 años. En relación con el NSE el 13,7\% de los/as participantes pertenecían a un nivel marginal o bajo inferior, el 31,6\% a un nivel bajo superior, el $28,8 \%$ a un nivel medio-bajo, el $18,6 \%$ a un nivel medio típico y el 7,3\% restante a un NSE medio alto o alto.

\subsection{Instrumentos de recolección de datos}

Variables Sociodemográficas: estas variables fueron incluidas para obtener información necesaria para la composición de las cuotas del muestreo; pero su rol en relación con las RS de la democracia argentina no forma parte de los objetivos del estudio. Para evaluar sexo, edad y nivel educativo se construyeron preguntas cerradas de alternativa fija. El índice para medir NSE contempla la relación entre cantidad de aportantes y miembros del hogar, la ocupación (tipo de trabajo, cantidad de personas a cargo o cantidad de empleados), nivel educativo y cobertura de salud del principal sostén del hogar (Comisión de Enlace Institucional, AAM-SAIMO-CEIM, 2006).

Representaciones Sociales de la Democracia Argentina: se aplicó la técnica de asociación de palabras que, por su carácter espontáneo y proyectivo, permite un acceso rápido y fácil a los elementos del universo semántico del objeto estudiado (Abric, 2001). En nuestro caso se brindó la consigna: ¿Qué se le viene a la mente si yo le digo: "democracia argentina"?; solicitando hasta 5 palabras o conceptos (evitando frases completas), dado que la literatura previa sugiere que esa es la cantidad de palabras que las personas son capaces de asociar espontáneamente (Wagner \& Hayes, 2005). Además, se pidió a los/as participantes que asignaran a cada una un signo en función de si la consideraban positiva $(+)$, negativa (-) o neutra (o).

Participación Política: se preguntó acerca de la participación (si/no y su frecuencia) en organizaciones políticas, sindicales y de la sociedad civil durante el último año (incluyendo organizaciones y grupos informales). Teniendo en cuenta que se toma como indicador de involucramiento político, sólo se analizaron como "participantes" a quienes lo hacían una vez por mes o más $(\mathrm{N}=62)$; excluyendo acciones participativas aisladas de baja intensidad $(\mathrm{N}=39)$, que tampoco fueron tomadas en cuenta en el grupo de no participantes $(\mathrm{N}=353)$.

Sofisticación Política: combina indicadores de conocimiento e interés político. Para evaluar conocimiento político se aplicó la escala validada localmente de Brussino, Sorribas y Medrano (2008) $(\alpha=, 76)$. Consta de 8 preguntas sobre conocimiento cívico y político (por ejemplo, nombre los tres poderes del estado y partido con mayor cantidad de miembros en el poder legislativo provincial). Las respuestas se categorizan en tres posiciones: $0=$ no sabe /incorrecta, $1=$ parcialmente correcta y $2=$ correcta. La sumatoria de respuestas da el puntaje total de la escala. Respecto del interés político, aplicamos la escala adaptada al contexto local por Brussino, Rabbia y Sorribas (2009). Plantea 6 situaciones vinculadas a la política a las cuales el/la participante debe responder indicando el nivel de interés que le generan, desde $1=$ ningún interés a $5=$ mucho interés $(\alpha=, 93)$. Los puntajes de ambas fueron estandarizados y combinados en un único indicador de SP. Para utilizarla como variable de clasificación fueron etiquetados como de "baja SP" (N=82) quienes puntuaban una desviación estándar o más por debajo de la media del grupo y, como de "alta SP" $(\mathrm{N}=85)$, quienes puntuaran una desviación estándar o más por encima. Se excluye aquí a personas con puntuaciones intermedias $(\mathrm{N}=283)$. 


\subsection{Procedimiento}

Entrevistadores capacitados aplicaron individualmente un cuestionario en formato papel. Previo a la administración se brindó información sobre los objetivos y alcance del estudio y se pidió consentimiento informado; garantizando la confidencialidad y anonimato de los datos obtenidos.

\subsection{Análisis de datos}

Se adoptó la perspectiva estructural de las RS, entendidas como sistema dual núcleo/periferia (Abric, 1976; citado en Moliner \& Abric, 2015): mientras que el núcleo central recupera elementos con consenso grupal amplio y relativa estabilidad (ligados a la historia del grupo, a aspectos ideológicos, valores y normas); los elementos periféricos son menos compartidos, están sujetos a cambios y cumplen una función defensiva del núcleo en su relación con el ambiente (Moliner \& Abric, 2015; Parales-Quenza \& Vizcaíno-Gutiérrez, 2007; Wachelke, 2012). Esto es relevante en tanto comprendemos que dos RS serán diferentes si estas diferencias alcanzan al nivel del núcleo. Previo al análisis, se editaron las evocaciones unificando singulares y plurales y género y se asignaron términos comunes para los sinónimos, prefiriendo la opción más frecuente. Luego, se calculó la cantidad de evocaciones totales, cantidad de palabras diferentes y de evocaciones promedio por participante y -para cada grupo- su connotación (porcentaje del total de evocaciones positivas, negativas y neutras). Además, estimamos el índice de polaridad intrasujetos (De Rosa, 2002) para conocer la connotación predominante para cada uno/a de ellos/as. Este índice surge de la resta de las evocaciones negativas que da una persona al total de positivas, dividido por el total de sus evocaciones. El resultado va de -1 a 1 , de modo que los valores entre $-1 /-, 50$ señalan RS negativas, entre -,40/,40 neutras y entre ,50/1 positivas (De Rosa, 2002). Teniendo en cuenta la dispersión en las palabras, se generaron categorías más inclusivas para realizar los análisis de estructura. Para ello, se siguió un procedimiento inductivo que conllevó la creación de categorías fundamentadas en los datos (Strauss \& Corbin, 2002), con revisión de dos jueces independientes. A continuación, se realizaron análisis de protipicidad con el software Iramuteq para conocer la estructura de las RS para cada grupo. Este procedimiento, propuesto por Vergés (1992), permite estimar la conformación del núcleo de la RS y la estructuración del resto de los elementos periféricos tomando en cuenta la cantidad de menciones y su orden de evocación. Así, se genera una matriz de 2 x 2 en la cual las categorías más evocadas y enunciadas primero (núcleo de la RS) se presentan en la casilla 1; aquellas que se evocan con un frecuencia igual o mayor a la media, pero en los últimos lugares conforman los elementos de la primera periferia; las que tienen una frecuencia inferior a la media, pero mencionadas en los primeros lugares conforman la zona de contraste y, finalmente, en la segunda periferia se encuentran evocaciones de baja frecuencia y bajo rango que enriquecen el campo semántico pero representan elementos idiosincráticos. Los puntos de corte para identificar los elementos de los cuadrantes en cada grupo son estimados en función de sus puntuaciones medias. 


\section{Resultados}

Sólo 9 participantes no pudieron generar ninguna evocación ( $2 \%$ del total), una proporción muy inferior al 25\% promedio que reportaron Dalton et al. (2007). En total, se registraron 1785 evocaciones, de las cuales 555 (31,09\%) fueron palabras o expresiones diferentes. De estas, 322 palabras fueron mencionadas sólo una vez (58,02\%), siendo el promedio de evocaciones por participante de 3,93 (sobre un máximo de 5).

Para el análisis prototípico se utilizaron las palabras distribuidas en las 69 categorías creadas a partir del análisis cualitativo. En primer lugar, comparamos el grupo que participaba políticamente con el que no. Como señalamos antes, el 77,7\% de la muestra no realizaba ninguna acción ( $\mathrm{N}=353)$; el 8,7\% participaba esporádicamente ( $\mathrm{N}=39)$ y el 13,6\% participaba con regularidad $(\mathrm{N}=62)$. Los análisis descriptivos muestran que quienes participaban reportaban una cantidad notablemente mayor de evocaciones promedio $(4,5)$ que quienes no participaban $(3,9)$ (Tabla 1). Esto puede entenderse como producto del conocimiento y familiaridad con el concepto (Dalton et al., 2007), pero también como indicador de la importancia que tiene para el grupo (Magioglou, 2000). Además, quienes participaban asociaron más términos positivos tanto en términos globales como intrasujetos.

Tabla 1. Indicadores descriptivos cuantitativos de las RS de personas que no participan $(\mathrm{N}=353)$ y personas que participan $(\mathrm{N}=62)$ políticamente

\begin{tabular}{ccc}
\hline & No Participantes & Participantes \\
\hline $\begin{array}{c}\text { Cantidad de Evocaciones (F) } \\
\text { Evocaciones Promedio por }\end{array}$ & 1378 & 278 \\
Participante & 3,9 & 4,5 \\
Connotación (\% sobre el total) & & \\
Negativa & & $38,13 \%$ \\
Neutra & $42,74 \%$ & $15,47 \%$ \\
Positiva & $17,49 \%$ & $46,4 \%$ \\
Índice de Polaridad Instra-sujetos (\%) & $39,77 \%$ & \\
Negativa & & $29 \%$ \\
Neutra & $32,6 \%$ & $35,5 \%$ \\
Positiva & $39,1 \%$ & $35,5 \%$ \\
\hline
\end{tabular}

Respecto de la estructura de la RS, en la Tabla 2 se exponen los resultados del grupo que no participaba políticamente. En función del tamaño de la muestra y dispersión de las evocaciones, se analizaron las categorías con 8 o más menciones (85,6\% del total). Los puntos de corte para la conformación de los cuadrantes fueron una frecuencia media de 25 y un rango medio (orden de evocación promedio de las categorías) de 2,61. 
Tabla 2. Contenido y Estructura de la RS de la democracia argentina en ciudadanos/as de Córdoba que no participan políticamente ( $\mathrm{N}=353)$.

\begin{tabular}{|c|c|c|c|c|c|c|}
\hline & Rango Medio $\leq 2$ & & & Rango Medio $>2$, & & \\
\hline & NÚCLEO & & & PRIMERA PERIFH & RIA & \\
\hline & & $\mathrm{F}$ & Rango & & $\mathrm{F}$ & Rango \\
\hline & Corrupción & 111 & 2,4 & Participación & 44 & 2,8 \\
\hline & Voto y Elecciones & 96 & 2,4 & Avance y Desarrollo & 38 & 3,3 \\
\hline & Libertad & 76 & 1,9 & Desigualdad & 33 & 3,2 \\
\hline 20 & $\begin{array}{c}\text { Libertad de información y } \\
\text { expresión }\end{array}$ & 47 & 2,4 & $\begin{array}{c}\text { Evocaciones negativas } \\
\text { inespecíficas }\end{array}$ & 31 & 2,7 \\
\hline.$\frac{\pi}{0}$ & Derecho & 42 & 2,6 & Injusticia & 28 & 2,8 \\
\hline 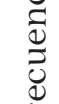 & $\begin{array}{c}\text { Gobierno y Sistema } \\
\text { Político }\end{array}$ & 40 & 2,4 & $\begin{array}{l}\text { Adjetivos negativos hacia } \\
\text { actores del sistema político }\end{array}$ & 28 & 3 \\
\hline ⿷匚⿳丨コ丨卜 & Adjetivos negativos & 37 & 2,6 & & & \\
\hline & $\begin{array}{c}\text { hacia el sistema político } \\
\text { argentino }\end{array}$ & & & Justicia & 28 & 2,8 \\
\hline & Igualdad & 35 & 2,1 & Valores & 27 & 2,7 \\
\hline & Sociedad y Ciudadanía & 29 & 2,5 & & & \\
\hline & ELEMENTOS CONTRA & STAI & JTES & SEGUNDA PERIFI & RIA & \\
\hline & & $\mathrm{F}$ & Rango & & $\mathrm{F}$ & Rango \\
\hline & Democracia Imperfecta & 22 & 2,1 & Mentira y engaño & 25 & 2,7 \\
\hline & $\begin{array}{c}\text { Referencias al } \\
\text { Radicalismo }^{1}\end{array}$ & 17 & 2,5 & Respeto y tolerancia & 20 & 3,7 \\
\hline & Inseguridad y delincuencia & 16 & 2,2 & Democracia delegativa & 20 & 3,1 \\
\hline & Patria, país y nación & 15 & 2,6 & Sistema económico & 19 & 3,1 \\
\hline & Poder del pueblo & 15 & 2,1 & Símbolos patrios & 17 & 3,1 \\
\hline & Soberanía & 15 & 2 & Referencias a dictaduras & 14 & 3 \\
\hline & Debilidad e inestabilidad & 12 & 2,1 & Opiniones y debate & 14 & 3,3 \\
\hline $\begin{array}{ll}20 \\
\text { ov } \\
\text { VI } \\
\text { a }\end{array}$ & Falsedad & 12 & 2,2, & $\begin{array}{l}\text { Otras referencias a } \\
\text { políticos }\end{array}$ & 13 & 3,3 \\
\hline 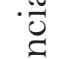 & Política & 12 & 2,6 & Violencia y conflicto & 13 & 3,4 \\
\hline$\stackrel{0}{0}_{0}^{0}$ & Decisiones & 10 & 2,3 & "No existe" & 12 & 2,7 \\
\hline 胥 & Adjetivos Positivos & 9 & 2,1 & Emociones positivas & 12 & 2,7 \\
\hline & Inmadurez del sistema & 8 & 1,8 & Poder & 11 & 3 \\
\hline & Referencias al peronismo ${ }^{2}$ & 8 & 2,5 & Unión & 11 & 2,8 \\
\hline & & & & $\begin{array}{l}\text { Recuperación de } \\
\text { democracia }\end{array}$ & 9 & 3,4 \\
\hline & & & & $\begin{array}{l}\text { Bienestar social y } \\
\text { económico }\end{array}$ & 9 & 3,3 \\
\hline & & & & Partidos políticos & 9 & 3,7 \\
\hline & & & & Poder Ejecutivo Nacional & 9 & 3,2 \\
\hline
\end{tabular}


Si bien en el núcleo de la RS se replican los elementos hallados en estudios previos relativos a la democracia liberal -específicamente: libertades, igualdad y derechos (Bruno \& Barreiro, 2015; Dalton et al., 2007; Moodie et al., 1995; Staerklé et al., 2015), se destacan otras dos categorías: corrupción y voto y elecciones. Esta última corresponde a una perspectiva procedimental restringida de la democracia, identificada con conceptualizaciones de baja sofisticación, propias de contextos de menor desarrollo democrático (Dalton et al., 2007). Por su parte, la noción de corrupción refiere a la dimensión del ser real de la democracia y lo hace negativamente. También conforman el núcleo las adjetivaciones negativas hacia el sistema político argentino, sosteniendo una RS de poco sofisticada en tanto son evocaciones inespecíficas con una valencia afectiva negativa fuerte. Por su parte, la noción de sociedad y ciudadanía es la única que remite a las bases sociales de la democracia, aunque no incluye referencias a su rol activo o como agente de cambio, sino que es una categoría descriptiva neutra (por ejemplo: colectivo, personas, ciudadanos). Es en la primera periferia donde se registran algunos elementos de definiciones sustantivas (justicia y -por su ausencia- injusticia y desigualdad) y de la dimensión prospectivo-ideal (participación, avance y desarrollo). Sin embargo, en este cuadrante también los contenidos asociados al ser real son negativos. Las menciones a instituciones del sistema, sus referentes y la política sólo son elementos de contraste (relevantes para un grupo pequeño) o de la segunda periferia (poco mencionados y poco relevantes).

A modo de síntesis, quienes no participaban políticamente sostuvieron RS poco sofisticadas, fuertemente ancladas en un ser real negativo y una noción de deber ser restringida, vinculada a principios liberales individualistas y procedimientos electorales. La posibilidad de cambio, el bienestar social y el rol ciudadano son secundarios y mencionados como carencia.

Para el caso de quienes participaban políticamente, al ser un grupo más pequeño, se incluyeron categorías con 2 o más menciones lo que permitió trabajar con un porcentaje similar del corpus al del grupo anterior $(85,25 \%)$. Los puntos de corte se establecieron en función de una frecuencia media de 6 y un rango medio de 2,72 (Tabla 3).

Tabla 3. Contenido y Estructura de la RS de la democracia argentina en ciudadanos/as de Córdoba que participan políticamente $(\mathrm{N}=62)$

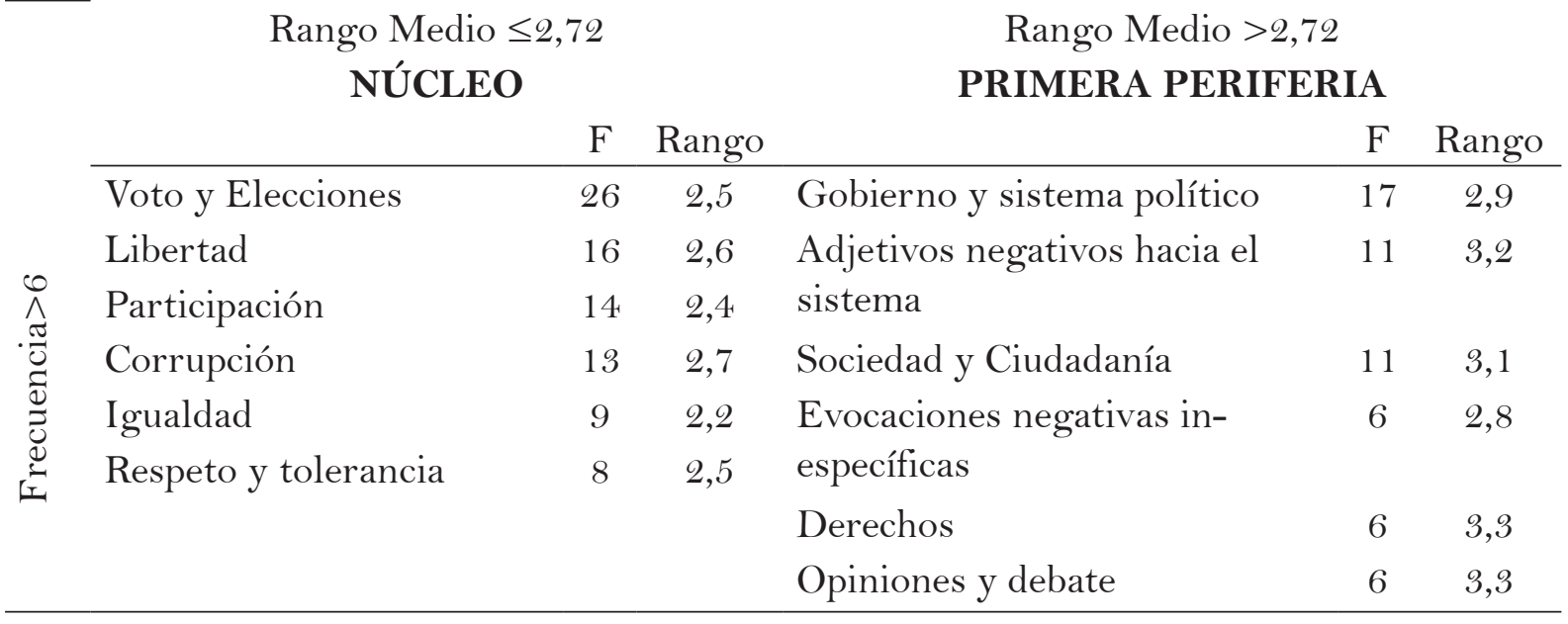




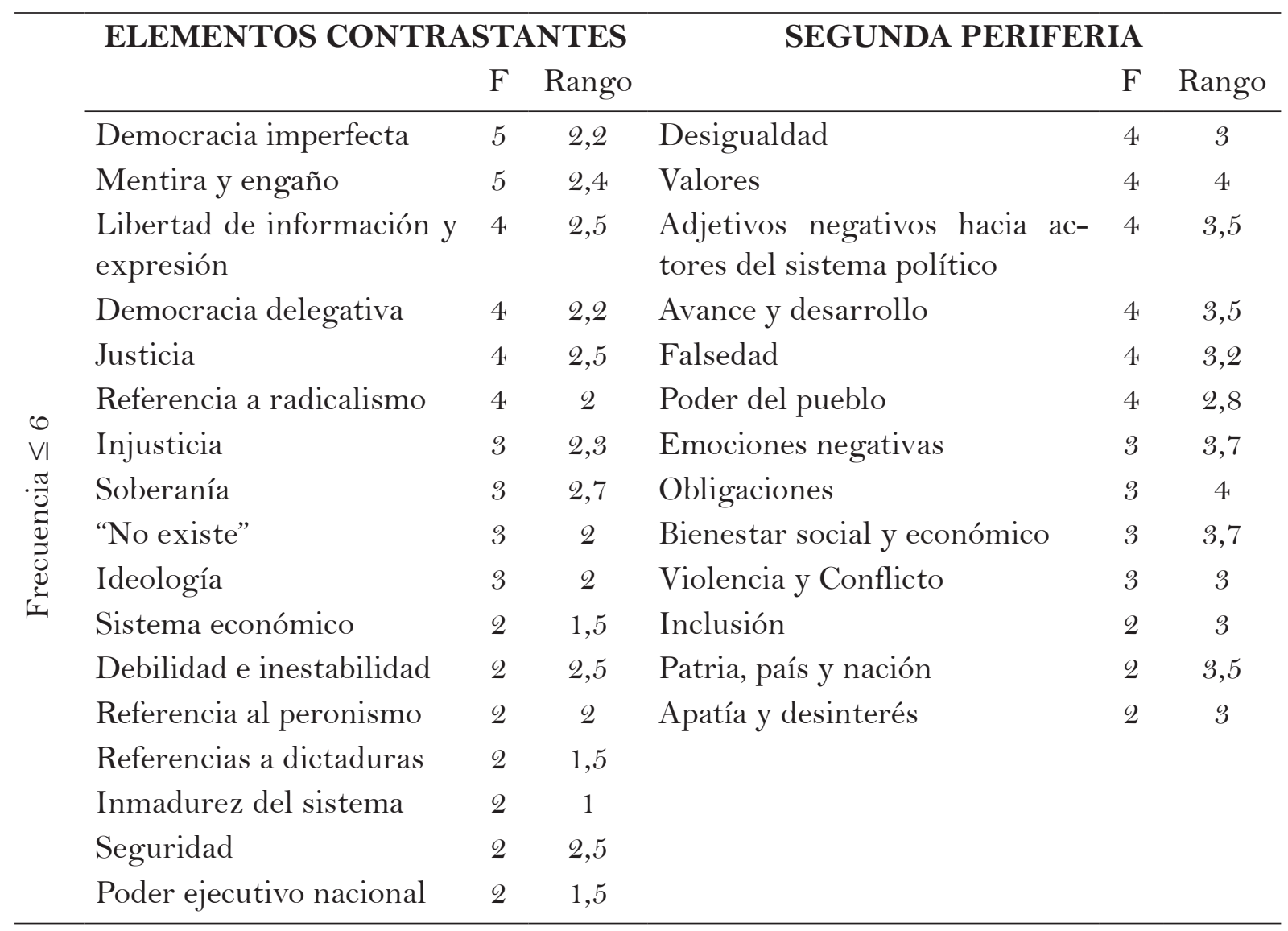

El núcleo comparte los elementos centrales de la democracia electoral y liberal del grupo que no participa. Sin embargo, las evocaciones negativas tienen menor presencia: mientras que en el grupo anterior la corrupción era la categoría de mayor peso, aquí es la cuarta más importante. Por su parte, conforman el núcleo la participación (acciones convencionales, no convencionales y de protesta) y el respeto y tolerancia (remite a valores colectivistas, al consenso, diálogo y aceptación), que se contraponen a perspectivas individualistas atadas a libertades personales. Además, en la primera periferia se recuperan nociones vinculadas a las opiniones y debate, ausentes en otros grupos. En este sentido, representan una valoración positiva del ser real democrático o-mínimamente- una visión prospectiva-ideal, señalando la capacidad de agencia para transformar la realidad negativa. Igualmente, entre los elementos periféricos surgen referencias negativas y menos sofisticadas del ser real. Finalmente -aún en un grupo de personas que participan- son escasas las referencias a los canales institucionales tradicionales de participación política (partidos políticos, poderes del estado y sus actores).

En resumen, el grupo que participa políticamente sostiene RS relativamente más sofisticadas, donde las percepciones negativas del ser real democrático son menos centrales y se destaca la capacidad de agencia ciudadana. Igualmente, hay pocas definiciones sustantivas y, en la dimensión ideal, aún prevalecen procedimientos electorales y valores de la democracia liberal. Sin embargo, las referencias a actores tradicionales del sistema representativo tienen poca presencia y predominan referencias a las bases sociales y canales no convencionales.

Complementariamente, evaluamos diferencias entre grupos de baja y alta SP. El número de evocaciones promedio por participante es significativamente más alto en el grupo 
de alta SP $(4,43)$, muy superior al del grupo de baja SP $(3,49)$ y similar al de grupo de personas que participan políticamente $(4,5)$ (Tabla 4). Respecto de la connotación de las evocaciones, las diferencias entre grupos son menos notables, siendo en el grupo de alta SP ligeramente más positivas. Más del $40 \%$ de estas son negativas en ambos grupos y menos del 30\% de los/as participantes dan definiciones positivas. Esta coexistencia entre términos positivos y negativos se replicó en todos los grupos y concuerda con lo señalado por la literatura (Cárdenas, Parra, Picón, Pineda, \& Rojas, 2007; Echebarría \& Álvarez, 1996; González Aguilar, 2016; Cerda et al., 2016; Yépez Hernández, 2003).

Tabla 4. Análisis descriptivos cuantitativos de RS sobre democracia en ciudadanos/as de baja $(\mathrm{N}=82)$ y alta $(\mathrm{N}=85)$ sofisticación política

\begin{tabular}{ccc}
\hline & Baja SP & Alta SP \\
\hline Cantidad de Evocaciones (F) & 286 & 377 \\
Evocaciones Promedio por Participante & 3,49 & 4,43 \\
Connotación (\% sobre el total) & & \\
Negativa & $46,92 \%$ & $40,68 \%$ \\
Neutra & $14,38 \%$ & $20,21 \%$ \\
Positiva & $38,7 \%$ & $39,11 \%$ \\
Índice de Polaridad Instra-sujetos (\%) & & \\
Negativa & $37,6 \%$ & $32,6 \%$ \\
Neutra & $37,6 \%$ & $39,5 \%$ \\
Positiva & $24,8 \%$ & $27,9 \%$ \\
\hline
\end{tabular}

Con relación al contenido de la RS, para el grupo de baja SP analizamos categorías con frecuencia igual o mayor a $2(90,17 \%$ del total). El punto de corte según orden de evocación promedio fue de 2,39 y la frecuencia media de 6,12. Este grupo recoge RS más homogéneas, con pocas categorías en el núcleo y primera periferia (Tabla 5). El núcleo remite tanto a los valores de la democracia liberal (libertad e igualdad) como a sus procedimientos (voto y elecciones). Además, se incluyen -más que en otros grupos- categorías de palabras negativas y poco sofisticadas (corrupción, evocaciones negativas inespecíficas, mentira y engaño). Las evocaciones negativas inespecíficas, en particular, son similares a las emociones negativas - por su valencia- pero conformadas principalmente de sustantivos con poca asociación directa con la democracia (por ejemplo: llanto, chiste, abismo, caos). De modo contrapuesto, en los elementos periféricos surgen asociaciones de la democracia sustantiva, no esperables en función de la baja implicación del grupo, pero que también están presentes en otros (como el desarrollo y la desigualdad). Por su parte, la zona de contraste recoge también muchas categorías negativas, coexistiendo con emociones positivas y algunas referencias a las bases sociales de la democracia (poder del pueblo, sociedad y ciudadanía, decisiones, valores). Las referencias a políticos y a la política, así como a las instituciones representativas están casi ausentes. 
Tabla 5. Contenido y Estructura de la RS de la democracia argentina en ciudadanos/as de Baja SP $(\mathrm{N}=82)$

\begin{tabular}{|c|c|c|c|c|c|}
\hline \multicolumn{3}{|l|}{ Rango Medio $\leq 2,39$} & \multicolumn{3}{|l|}{ Rango Medio >2,39 } \\
\hline \multicolumn{3}{|l|}{ NÚCLEO } & \multicolumn{3}{|c|}{ PRIMERA PERIFERIA } \\
\hline & $\mathrm{F}$ & Rango & & $\mathrm{F}$ & Rango \\
\hline Corrupción & 28 & 2,2 & Desigualdad & 11 & 3,6 \\
\hline Voto y elecciones & 26 & 2,2 & Libertad de información y & 11 & 2,6 \\
\hline Libertad & 20 & 1,9 & expresión & & \\
\hline Evocaciones negativas & 10 & 1,9 & Injusticia & 10 & 2,8 \\
\hline inespecíficas & & & Avance y desarrollo & 9 & 2,9 \\
\hline Mentira y engaño & 10 & 2,3 & Derechos & 7 & 2,6 \\
\hline Igualdad & 8 & 1,4 & & & \\
\hline \multicolumn{3}{|c|}{ ELEMENTOS CONTRASTANTES } & \multicolumn{3}{|c|}{ SEGUNDA PERIFERIA } \\
\hline & $\mathrm{F}$ & Rango & & $\mathrm{F}$ & Rango \\
\hline \multirow{2}{*}{$\begin{array}{c}\text { Adjetivos negativos hacia el } \\
\text { sistema }\end{array}$} & \multirow{2}{*}{6} & \multirow{2}{*}{2,2} & Inseguridad y delincuencia & 6 & 2,5 \\
\hline & & & Participación & 6 & 3,3 \\
\hline Valores & 5 & 1,8 & Violencia y conflicto & 4 & 3 \\
\hline \multirow{2}{*}{$\begin{array}{c}\text { Gobierno y sistema político } \\
\text { "No existe" }\end{array}$} & 5 & 2,2 & \multirow{2}{*}{$\begin{array}{l}\text { Adjetivos negativos hacia } \\
\text { actores del sistema }\end{array}$} & \multirow{2}{*}{4} & \multirow{2}{*}{3,8} \\
\hline & 4 & 1,3 & & & \\
\hline Soberanía & 4 & 1,5 & Democracia delegativa & 3 & 2,7 \\
\hline Otras referencias a políticos & 4 & 2 & Patria, país y nación & 3 & 2,7 \\
\hline Justicia & 4 & 2 & Crisis & 3 & 2,7 \\
\hline Emociones positivas & 4 & 2 & Obligaciones & 3 & 3 \\
\hline Poder del pueblo & 4 & 2 & Respeto y tolerancia & 3 & 3,3 \\
\hline Poder Ejecutivo Nacional & 4 & 2,2 & Referencia al Kirchnerismo & 2 & 3 \\
\hline Democracia imperfecta & 3 & 1,5 & Utopía & 2 & 3 \\
\hline Sistema económico & 3 & 2 & Símbolos patrios & 2 & 4 \\
\hline Adjetivos positivos & 3 & 2 & Bienestar social y económico & 2 & 4 \\
\hline Decisiones & 3 & 2 & Opiniones y debate & 2 & 4,5 \\
\hline Falsedad & 3 & 2 & & & \\
\hline Sociedad y ciudadanía & 3 & 2 & & & \\
\hline Unión & 2 & 2 & & & \\
\hline
\end{tabular}

Así, consistentemente con su baja implicación, la RS en este grupo es particularmente poco sofisticada e inespecífica, definida -en su dimensión ideal-principalmente en términos de libertades y procedimientos, con fuerte énfasis en su vulneración en la dimensión real; sin embargo, no reconoce como central ningún actor formal e informal de la política ni la capacidad de agencia de la ciudadanía en ese marco -o lo hace escasamente-.

Para el caso del grupo de alta SP también se estimó el análisis de prototipicidad sobre las categorías con frecuencia igual o mayor a 2 (88,71\% del corpus). El orden de evocación promedio fue de 2,73 y la frecuencia media de 6,38. El núcleo en este grupo es notablemente más heterogéneo, denotando que su nivel de conocimiento e información no conlleva 
la reproducción de un discurso institucionalizado y hegemónico, de carácter ideológico, como se daría en grupos con formación específica (Ruiz \& Coy, 2015).

Tabla 6. Contenido y Estructura de la RS de la democracia argentina en ciudadanos/as de Alta SP $(\mathrm{N}=85)$

\begin{tabular}{ccccccc}
\hline \multicolumn{2}{c}{ Rango Medio $\leq 2,73$} & \multicolumn{5}{c}{ Rango Medio $>2,73$} \\
\hline NÚCLEO & & \multicolumn{3}{c}{ PRIMERA PERIFERIA } \\
& F & Rango & F & Rango \\
\hline Corrupción & 23 & 2,6 & Voto y elecciones & 28 & 2,9 \\
Participación & 21 & 2,4 & Adjetivos negativos hacia el & 14 & 2,9 \\
Libertad & 16 & 2 & sistema & & \\
Gobierno y sistema político & 13 & 2,7 & Sistema económico & 9 & 3 \\
Igualdad & 10 & 2 & Avance y desarrollo & 9 & 3,9 \\
Sociedad y ciudadanía & 10 & 2,2 & Derechos & 7 & 3,1 \\
Poder del pueblo & 8 & 2,1 & Injusticia & 7 & 2,9 \\
Referencia al radicalismo & 8 & 2,1 & & & \\
Adjetivos negativos hacia & 8 & 2,6 & & & \\
actores del sistema & & 2,6 & & & \\
Democracia delegativa & 8 & 2,6 & & \\
Democracia imperfecta & 7 & 1,6 & & & \\
\hline
\end{tabular}

\section{ELEMENTOS CONTRASTANTES}

SEGUNDA PERIFERIA

\begin{tabular}{|c|c|c|c|c|c|}
\hline & $\mathrm{F}$ & Rango & & $\mathrm{F}$ & Rango \\
\hline Libertad de información y & 6 & o & Otras referencias a políticos & 6 & 4,5 \\
\hline expresión & 6 & 2 & Respeto y tolerancia & 5 & 2,8 \\
\hline Inmadurez del sistema & 6 & 1,3 & Mentira y Engaño & 5 & 2,8 \\
\hline Referencia al kirchnerismo & 6 & 2,7 & Símbolos patrios & 5 & 3 \\
\hline Justicia & 6 & 2,7 & Falsedad & 5 & 4 \\
\hline Referencias a dictaduras & 5 & 2,5 & Opiniones y debate & 5 & 4 \\
\hline Ideología & 4 & 2,5 & Desigualdad & 4 & 2,8 \\
\hline Debilidad e inestabilidad & 4 & 2,5 & Poder & 4 & 2,8 \\
\hline Soberanía & 3 & 1,3 & Apatía y desinterés & 4 & 3 \\
\hline "No existe" & 3 & 1,7 & Evocaciones negativas inespe- & & \\
\hline Inseguridad y delincuencia & 3 & 1,7 & cíficas & 4 & 3,2 \\
\hline Referentes de la historia & 2 & 1 & Patria, país y nación & 4 & 4 \\
\hline Poder Ejecutivo Nacional & 2 & 1 & Recuperación de la democracia & 4 & 4,2 \\
\hline Medios de comunicación & 2 & 2 & Crisis & 3 & 3,3 \\
\hline \multirow[t]{9}{*}{ Seguridad } & 2 & 2,5 & Abuso de poder & 3 & 4 \\
\hline & & & Importancia de la democracia & 3 & 4,7 \\
\hline & & & Estilo de vida & 2 & 3 \\
\hline & & & Valores & 2 & 3,5 \\
\hline & & & Obligaciones & 2 & 4 \\
\hline & & & Unión & 2 & 4 \\
\hline & & & Equidad & 2 & 4 \\
\hline & & & Bienestar social y económico & 2 & 4,5 \\
\hline & & & Violencia y conflicto & 2 & 4,5 \\
\hline
\end{tabular}


Respecto del contenido, los elementos de la democracia liberal representativa resultan predominantes también en este grupo, tanto en términos teóricos/ideales como concretos. Así, esta RS se asienta fuertemente sobre la idea de gobierno y sistema político, incluyendo referencias directas a delegación del poder en los representantes (democracia delegativa) y a uno de los partidos políticos tradicionales (referencias al radicalismo). Es el único grupo donde elementos de la política tradicional son centrales. Además, se recogen en el núcleo los mismos valores mencionados en otros (libertad e igualdad), pero no el voto y elecciones, que aquí son periféricos. Si bien algunos elementos negativos son nucleares, sobre todo la corrupción, también hay elementos que señalan la capacidad de agencia de la ciudadanía. Por ejemplo, la idea de poder del pueblo sólo conforma el núcleo representacional en este grupo; a la que se suma la participación y referencias a la sociedad y ciudadanía. Respecto del contenido periférico, además del voto y las elecciones, se registran referencias negativas relativas al ser real, incluyendo referencia al sistema económico (neoliberalismo, capitalismo, oligarquía). Por su parte, la zona de contraste es heterogénea; destacándose la ideología, ausente en la mayoría de los grupos, así como medios de comunicación y soberanía.

A modo de síntesis, este grupo es el que tiene RS más diferentes de los otros, siendo además más heterogéneas. Es el único en que el voto no es central, aunque recoge elementos procedimentales de la democracia representativa más sofisticados. Igualmente, la democracia liberal es central, pero con mayor protagonismo ciudadano y menos definiciones negativas.

\section{Conclusiones}

En términos generales, los resultados expuestos coinciden con la literatura previa, replicando la centralidad de definiciones liberales de democracia: cuando piensan en democracia la mayoría de las personas lo hacen en función de valores liberales y desde una perspectiva individualista y pasiva (Dalton et al., 2007; Magioglou, 2000; Moodie et al., 1995; Staerklé et al., 2015; Yépez Hernández, 2003; Zovatto, 2002). Más específicamente, la libertad, igualdad y corrupción conforman el núcleo representacional en todos los grupos y el voto en tres de ellos. Además, en un sistema presidencialista como el argentino, esa figura no fue central; como tampoco lo fueron los partidos políticos e instituciones y procesos vinculados a la noción de gobierno, poder, política y deliberación. Esto puede interpretarse como evidencia del desencanto con la política y lo político señalado en los antecedentes (Bruno \& Barreiro, 2015; Corporación Latinoabrómetro, 2015). Además, esperábamos que los grupos más involucrados políticamente brindasen definiciones sustantivas, señalando el rol del sistema político respecto de temas como la justicia social, el bienestar y la ampliación de derechos (Bermúdez et al., 2004; Crow, 2010), Sin embargo, este contenido tuvo apenas un rol periférico y no permitió diferenciar entre grupos. En esta línea, la definición ideal o abstracta de democracia no parece ser un concepto en disputa. Así, en todos los grupos predominaron evocaciones menos específicas, poco sofisticadas y de fuerte carga afectiva -principalmente negativa-. Sin embargo, esta carga negativa de las evocaciones remitía mayormente a un nivel concreto y no atacaba la legitimidad de la democracia como principio normativo; siendo consistente con la tradición de estudios que diferencian el apoyo o legitimación de un sistema político y la satisfacción con su desempeño (Dalton 2004; Dalton et al., 2007; Dalhberg et al., 2015). 
Más allá de la descripción de los aspectos comunes, el aporte central de nuestro trabajo fue complejizar la comprensión de las RS a partir del análisis del rol del involucramiento político. En esta línea, los grupos de personas políticamente más implicadas no sólo tendían a mostrar RS más positivas que las no implicadas; sino que evocaban más palabras, más específicas y con mayor contenido político. Por ejemplo, el grupo de alta SP fue el único en el que se aludía frecuentemente a procedimientos y actores centrales del sistema político representativo (gobierno y sistema político, delegación, partidos políticos), denotando mayor conocimiento de su funcionamiento; lo cual podría señalar que el mayor conocimiento cívico y político de este grupo efectivamente contribuye a estas expresiones. En esta línea, el carácter de la medición de sofisticación política -con una orientación hacia elementos de la democracia representativa- puede haber condicionado este tipo de expresiones. Por esta razón, resultaría pertinente contemplar otras aproximaciones a la SP que excedan el ámbito de la política institucionalizada o tradicional y permitan complejizar el entendimiento del rol de esta variable.

Por su parte, quienes participaban políticamente -si bien también tenían alta implicación política- no evocaban procesos e instituciones tradicionales y, en ello, no se diferenciaban del resto de los grupos. Sin embargo, este grupo mostraba una mayor identificación de la capacidad de agencia de la ciudadanía que no era vehiculizada a través de las vías convencionales de ejercicio de la política. Así, si bien ambos grupos de mayor implicación producían RS relativamente más complejas o sofisticadas, la SP se asoció más al reconocimiento de los procedimientos e instituciones de la democracia representativa, mientras que la participación -en consonancia con lo que proponían Tessler y Gao (2008)- parecía operar más como agente socializador a partir de la experiencia organizativa en términos de valores (respeto y tolerancia) y del reconocimiento de las bases sociales de la democracia. Finalmente, si bien en ningún grupo se encontraron ausentes las valoraciones negativas de la democracia real, es en los dos grupos de mayor implicación donde ésta tiene un protagonismo relativamente menor y en los que puede identificarse una dimensión prospectivo-ideal (Lozada, 1997). De este modo, se reconoce un potencial de cambio del sistema político con algún nivel de protagonismo ciudadano; siendo los únicos en los que la participación es central y -además- refieren al poder del pueblo o al respeto y la tolerancia.

A modo de síntesis, señalamos que este estudio nos permitió dar cuenta de RS de la democracia que, en general, tienen un contenido hegemónico, inespecífico y poco sofisticado, denotando poco reconocimiento o relevancia de sus atributos procedimentales no electorales y del rol de la sociedad civil. En esta línea, si bien era esperable que los elementos del núcleo central tuviesen cierto grado de inespecificidad -lo cual les permite una mayor capacidad asociativa con elementos de la periferia (Wachelke, 2012)- su carácter fue cualitativamente diferente entre grupos. Como mencionamos, el distanciamiento de lo político se reflejaba en RS más pesimistas que asignaban un rol limitado o nulo a la ciudadanía, mientras que en los grupos de alta implicación este carácter -al menos parcialmente- se revertía: al tiempo que en la mayoría de la ciudadanía predominaba una visión "apolítica”, donde el poder no era una categoría en disputa y la deliberación estaba prácticamente ausente; la implicación política se asociaba con la evocación de algunos contenidos no hegemónicos relativos al rol de la ciudadanía. No se desprende de ello una lectura normativa de las RS de la democracia, sino que se pretende enfatizar en el rol que potencialmente tiene la experiencia organizativa como espacio de socialización y acción política, vehículo de representaciones que priorizan el protagonismo ciudadano a través de aspectos vinculados 
al capital social (énfasis en la convivencia y la tolerancia, además de la participación). En este sentido, la confianza interpersonal y la tolerancia política fortalecen las bases sociales de la democracia y se relacionan con la confianza institucional y política, la satisfacción y el apoyo a la democracia (Dalton, 2004; Inglehart, 2003; Norris, 2011; Zmerli \& Newton, 2008). Consecuentemente, sería relevante profundizar en el rol de los procesos y agencias de socialización política en el desarrollo de las RS y sus correlatos actitudinales y comportamentales; ya sea en estudios longitudinales o a través de programas de formación cívica específicos.

Por otra parte, es relevante abordar algunas limitaciones que se desprenden del diseño metodológico del presente estudio. En primer lugar, como se mencionó anteriormente, sería relevante incorporar en posteriores estudios un análisis de la sofisticación política que exceda la política tradicional. Además, la diferencia en el tamaño de los grupos -con una muestra heterogénea de personas que no participan políticamente contra grupos más pequeños de personas políticamente involucradas- representa una limitación condicionada por las características de la participación política en el contexto analizado. En esta línea, sería relevante contar con una muestra más amplia de personas que participen y/o abordajes cualitativos complementarios que permitan evaluar la interacción entre los distintos aspectos de la implicación política. Finalmente, en el marco de una perspectiva de cultura política de la legitimidad, es relevante examinar la relación entre esta y las expectativas hacia la democracia expresadas en las RS; identificando formas de vinculación con el sistema político en función de aspectos valorativos, ideológicos y comportamentales. El objetivo último es aportar a la comprensión de las bases psicosociales de la desafección política y sus consecuencias para el desarrollo y calidad de las democracias en la región.

\section{Notas}

1 Uno de los partidos tradicionales del sistema político argentino al que pertenecía Raúl Alfonsín, primer presidente democrático luego de la última dictadura militar y señalado como el "padre de la democracia".

2 Refiere al Partido Justicialista, otro de los partidos tradicionales del sistema político argentino, al que pertenecieron la mayoría de los presidentes democráticos desde su creación.

\section{Referencias}

Abric, J. C. (2001). Metodología de recolección de las representaciones sociales. En Prácticas sociales y representaciones (pp. 53-74). México, DF: Presses Universitaires de France. Ediciones Coyoacán.

Bermúdez, M. N., Savino, L. D., \& Zenklussen, L. A. (2004). Representaciones sobre democracia y participación en la juventud de la ciudad de Córdoba. Cuadernos de la Facultad de Humanidades y Ciencias Sociales. Universidad Nacional de Jujuy, 22, 129-150.

Bruno, D. \& Barreiro, A. (2015). La representación social de la democracia de adolescentes argentinos. Escritos de Psicología, 8(3), 33-40. 
Brussino, S., Rabbia, H. H., \& Sorribas, P. (2009). Perfiles sociocognitivos de la participación política de los jóvenes. Interamerican Journal of Psychology, 43(2), 279-287.

Brussino, S., Sorribas, P., \& Medrano, L. (2008). Características psicométricas de la medición del conocimiento político. Revista Iberoamericana de diagnóstico y evaluación psicológica, 25(1), 179-192.

Cárdenas, M., Parra, L., Picón, J., Pineda, H., \& Rojas, R. (2007). Las representaciones sociales de la política y la democracia. Ultima década, 15(26), 53-78.

Cerda, Ó. R., Cadena Herrera, C. A., Saldívar Moreno, D. M., \& Mendoza Romero, A. (2016). Representación social de la democracia: de lo ideal a lo real. Revista Polis, 1(2), 33-56.

Comisión de Enlace Institucional AAM-SAIMO-CEIM (2006). NSE 2006. Antecedentes, marco conceptual, enfoque metodológico y fortalezas. Buenos Aires: Author.

Corporación Latinobarómetro. (2015). Informe 1995-2015. Santiago, Chile: Corporación Latinobarómetro.

Crow, D. (2010). The party's over: citizen conceptions of democracy and political dissatisfaction in Mexico. Comparative Politics, 43(1), 41-61.

Dahllberg, S., Linde, J., \& Holmberg, S. (2015). Democratic discontent in old and new democracies: Assessing the importance of democratic input and governmental output. Political Studies, 63(S1), 1837.

Dalton, R. J. (2004). Democratic challenges, democratic choices. Oxford: Oxford University Press.

Dalton, R. J., Sin, T. C., \& Jou, W. (2007). Understanding democracy: Data from unlikely places. Journal of Democracy, 18(4), 142-156.

De Rosa, S. A. (2002). The associative network: a technique for detecting structure, contents, polarity and stereotyping indexes of the semantic fields. European review of applied psychology, 52(3-4), 181-200.

Echebarría, A. \& Álvarez, J. (1996). Representaciones sociales de la democracia y el sistema electoral: estudio comparativo entre México y el País Vasco. Revista de psicología social, 11(1), 47-69.

González-Aguilar, F. (2016). Representaciones sociales de la democracia en estudiantes universitarios: avances y claves conceptuales. Revista Mexicana de Orientación Educativa, 13(31), 12-21.

Hernández Sampieri, R., Fernández Collado, C., \& Baptista Lucio, P. (1991). Metodología de la Investigación. México: MacGraw-Hill.

Inglehart, R. (2003). How solid is mass support for democracy - and how can we measure it? PS: Political Science \& Politics, 36(1), 51-57.

Jodelet, D. (2011). Returning to past features of Serge Moscovici's theory to feed the future. Papers on Social Representations, 20, 1-39.

Lavine, H. \& Gschwend, T. (2007). Issues, party and character: The moderating role of ideological thinking on candidate evaluation. British Journal of Political Science, 37(1), 139-163.

Lozada, M. (1997). Democracia y representaciones: la cuestión de lo social. Fermentum, 7(20), 93-106.

Magioglou, T. (2000). Social representations of democracy; Ideal versus reality. A qualitative study with young people in Greece. Paris: Maison des Sciences de l'Homme.

Moliner, P. \& Abric, J. C. (2015). Central Core Theory. En G. Sammut, E. Andreouli, G. Gaskell, \& J. Valsiner (Eds.), The Cambridge Handbook of Social Representations (pp. 83- 95). Cambridge: Cambridge University Press. 
Moodie, E., Marková, I., \& Plichtova, J. (1995). Lay representations of democracy: A study in two cultures. Culture E Psychology, 1(4), 423-453.

Muraro, O. (2012). El NSE en la encuesta anual de hogares urbanos (INDEC). Sociedad Argentina de Investigadores de Marketing y Opinión. Disponible en http://www.saimo.org.ar/archivos/observatoriosocial/NSE-en-la-EAHU.pdf

Norris, P. (2011). Democratic deficit: Critical citizens revisited. Cambridge: Cambridge University Press.

Parales-Quenza, C. J. \& Vizcaíno-Gutiérrez, M. (2007). Las relaciones entre actitudes y Representaciones sociales: Elementos para una integración conceptual. Revista Latinoamericana de Psicología, $39(2), 351-361$.

Pérez, M. P. (2003). A propósito de las representaciones sociales: apuntes teóricos, trayectoria y actualidad. CD Caudales, CIPS. Disponible en http://biblioteca.clacso.edu.ar/Cuba/cips/20130628110808/ Perera perez repr sociales.pdf

Rhee, J. W. \& Capella, J. N. (1997). The role of political sophistication in learning from news: Measuring schema development. Communication Research, 24(3), 197-233.

Ruiz, J. I. \& Coy, A. (2004). Esquemas cognitivos de base, contenido semántico y estructura de las representaciones sociales de la democracia. Acta Colombiana de Psicología, 12, 5-17.

Ruiz, J. I., \& Coy, A. (2015). Esquemas cognitivos de base, contenido semántico y estructura de las representaciones sociales de la democracia. Acta Colombiana de Psicología, 12, 5-17.

Staerkle, C., Falomir-Pichastor, J. M., Pereira, A., Berent, J., \& Butera, F. (2015). Global value perceptions: The legitimising functions of western representations of democracy. European Journal of Social Psychology, 45(7), 896-906.

Strauss, A. \& Corbin, J. (2002), Bases de la investigación cualitativa. Técnicas y procedimientos para desarrollar la teoria fundamentada. Medellín, COL: Editorial Universidad de Antioquia.

Strauss Tessler, M. \& Gao, E. (2008). Democracy and the political culture orientations of ordinary citizens: a typology for the Arab world and beyond. International Social Science Journal, 59(192), 197207.

Torres Stock1, C. M. \& Zubieta, E. M. (2015). Consenso y divergencias en las representaciones sociales de la dirigencia política. Revista de Psicología (PUCP), 33(1), 87-129.

Vergès, P. (1992). L'evocation de l'argent: Une méthode pour la définition du noyau central d'une représentation. Bulletin de Psychologie, 45(405), 203-209.

Wachelke, J. (2012). Social Representations: A Review of Theory and Research from the Structural Approach. Universitas Psychologica, 11(3), 729-741.

Wagner, W. \& Hayes, N. (2005). Everyday Discurse and Common Sense. The theory of Social Representations. New York: Palgrave Macmillan.

Weisberg, H. F. \& Nawara, S. P. (2010). How sophistication affected the 2000 presidential vote: Traditional sophistication measures versus conceptualization. Political Behavior, 32(4), 547-565.

Yépez Hernández, M. (2003). Representaciones sociales de democracia en estudiantes universitarios de la UNAM. Investigación de la comunicación. México en los albores del siglo XXI, 123-139

Zmerli, S. \& Newton, K. (2008). Social trust and attitudes toward democracy. Public opinion quarterly, 72(4), 706-724.

Zovatto, D. (2002). Valores, percepciones y actitudes hacia la democracia: una visión comparada latinoamericana, 1996-2002. América Latina Hoy, 32, 29-53. 


\section{DANIELA ALONSO \\ http://orcid.org/0000-0003-2080-9571}

Doctora en Psicología, Universidad Nacional de Córdoba. Becaria Posdoctoral CONICET. Miembro del equipo docente de la asignatura Psicología Política (Facultad de Psicología, UNC). Instituto de Investigaciones Psicológicas (CONICET y UNC), Argentina.

Dirección: Enfermera Gordillo esq. Enrique Barros. Ciudad Universitaria, Córdoba, Argentina. CP 5000

E-mail: dalonso@unc.edu.ar

\section{SILVINA BRUSSINO}

\section{http://orcid.org/0000-0002-1087-644X}

Doctora en Psicología, Universidad Nacional de Córdoba (UNC). Miembro de la Carrera de Investigador Científico y Tecnológico de CONICET (Investigadora Independiente), Docente Investigadora Categoría I de Universidades Nacionales. Directora de la Carrera de Doctorado en Psicología, UNC (2015-2018). Directora de la Carrera de Maestría en Metodología de la Investigación en Ciencias del Comportamiento, UNC (20152018). Miembro titular del Consejo Directivo del CIECS-CONICET (2015-2019) y del Honorable Consejo Directivo de la Facultad de Psicología (2014-2016; 2016-2018). Profesora Titular de la Cátedra Metodología de la Investigación Psicológica, Facultad de Psicología, UNC. Profesora Titular de la Cátedra Psicología Social, Facultad de Ciencias de la Comunicación, UNC. Profesora del Seminario electivo de Psicología Política, Facultad de Psicología, UNC. Instituto de Investigaciones Psicológicas [CONICET y UNC], Argentina.

E-mail: sbrussino@,conicet.gov.ar

\begin{tabular}{|c|c|}
\hline Histórico & $\begin{array}{l}\text { Submissão: 15/03/2018 } \\
\text { Revisão: 27/03/2019 } \\
\text { Aceite: 22/04/2019 }\end{array}$ \\
\hline $\begin{array}{l}\text { Contribuição dos } \\
\text { autores }\end{array}$ & $\begin{array}{l}\text { Concepção: AD; BS } \\
\text { Coleta de dados: AD; BS } \\
\text { Análise de dados: AD; BS } \\
\text { Elaboração do manuscrito: AD; BS } \\
\text { Revisões de conteúdo intelectual importante: AD; BS } \\
\text { Aprovação final do manuscrito: AD, BS }\end{array}$ \\
\hline Financiamento & Não houve \\
\hline
\end{tabular}

